\title{
Corrigendum
}

\section{Multi-netclust: an efficient tool for finding connected clusters in multi-parametric networks}

\author{
Arnold Kuzniar, Somdutta Dhir, Harm Nijveen, Sándor Pongor and Jack A.M. Leunissen \\ Bioinformatics (2010) 26(19) 2482-2483
}

The authors of the above article would like it to be known that the principle of Multi-netclust is incorrectly illustrated in Figure 1. The connected clusters shown in B and C do not correspond with the aggregation rules/formulae indicated below the illustrations. Swapping the clusters with one another restores this correspondence. The authors apologize for this error. 